\title{
Berechnung der Neutronenemission eines mit einer Hohlpinchentladung erzeugten D-T-Plasmas
}

\author{
R. G. A. Frank und R. Klingelhöfer
}

Institut für Kernverfahrenstechnik der Universität und des Kernforschungszentrums Karlsruhe

(Z. Naturforsch. 24 a, 1632-1636 [1969] ; eingegangen am 1. August 1969)

\begin{abstract}
The number of neutrons per discharge which are emitted during the collapse of a hollow D-T $z$-pinch is calculated. In the system of differential equations describing the radial acceleration of the cylinder by Lorentz-forces the internal pressure of the sheath is incorporated. Due to the pressure-term it is possible to calculate the time dependance of density, temperature and reaction-rate within the sheath. Using a capacitor bank of $200 \mathrm{~kJ}$ oule at $100 \mathrm{kV}$ and a hollow cylinder of $30 \mathrm{~cm}$ initial radius and $10 \mathrm{~cm}$ height the emission of $3 \cdot 10^{13}$ neutrons is expected during the implosion phase, lasting a few nsec. The optimization of the experimental parameters for maximum neutron emission is given.
\end{abstract}

Zur Lösung kerntechnischer und kernphysikalischer Probleme werden häufig intensive Neutronenimpulse benötigt. Solche „Neutronenblitze“ lassen sich mit verhältnismäßig geringem Aufwand unter Ausnutzung der Kernfusionsreaktion

$$
\mathrm{D}+\mathrm{T} \rightarrow \mathrm{He}^{4}+\mathrm{n}
$$

erzeugen.

Bei einem seit längerer Zeit benutzten Verfahren werden elektrisch beschleunigte Deuteronen kurzzeitig auf ein mit Tritium beladenes Target geschossen. Entsprechende Apparaturen liefern Momentanraten in der Größenordnung von $10^{14}$ Neutronen pro Sekunde, denen bei einer Impulsdauer von $10^{-6}$ bis $10^{-5}$ sec $10^{8}$ bis $10^{9}$ Neutronen pro Puls entsprechen ${ }^{1,2}$.

Merklich höhere Werte wurden kürzlich bei dem Plasma-Focus-Experiment von MATHER ${ }^{3}$ erreicht. Er benutzt eine aus zwei Koaxialleitern bestehende Plasmakanone, die am Ende ihres Innenleiters durch radialen Kollaps des axial beschleunigten Plasmaringes kurzfristig ein besonders dichtes und heißes Plasma erzeugt. Die mit einer Kondensatorenergie von $36 \mathrm{~kJ}$ erzielte Momentanrate beträgt $2 \cdot 10^{18}$ Neutronen pro Sekunde bei einer Impulsdauer von

Sonderdruckanforderungen erbeten an Dr. R. KLINGELHöFER, Institut für Kernverfahrenstechnik der Technischen Hochschule Karlsruhe, D-7500 Karlsruhe, Postfach 947.

1 W. Eyrich u. A. SchmidT, Two Compact High Intensity Pulsed Neutron Souces, KFK 304 [1965].

2 Conf. 660 925, Intense Neutron Sources, S. 581 und 589 [1966].

3 J. W. Mather, Plasma Phys. and Thermonucl. Reactions, Proc. of Culham Conf., Vol. II, S. 389 [1965]. - J. W. Mather, P. J. Bottoms u. H. H. Williams, Proc. APS Topical Conf. on Pulsed High Density Plasmas, C 1, LA 3770 [1967].
$1,5 \cdot 10^{-7}$ sec. Die Gesamtzahl der pro Puls emittierten Neutronen ist also $3 \cdot 10^{11}$.

Eine weitere Möglichkeit zur Erzeugung hoher Neutronenblitzraten bietet die von LINHART ${ }^{4}$ vorgeschlagene Hohlpinchanordnung. Bei diesem Experiment wird eine Hohlzylinder-förmige Plasmakonfiguration im Hochvakuum erzeugt, die unter der Wirkung des Magnetfeldes eines axialen Entladungsstromes in radialer Richtung kollabiert. In unmittelbarer Umgebung der Zylinderachse entsteht kurzfristig ein besonders heißes und dichtes Plasma.

Die Hohlpinchmethode wurde von MaIsonNIER, HAEgi und LinHart ${ }^{5}$ unter Verwendung von $\mathrm{Li} \mathrm{Al} \mathrm{D}_{4}$-Pulver als Ausgangsmaterial für das Plasma mit einer $50 \mathrm{~kJ}$-Kondensatorbatterie erprobt. Das Pulver konnte unter Ausnutzung elektrostatischer Effekte in Form eines Hohlzylinders in die Entladungskammer injiziert werden. Die Gesamtzahl der pro Puls emittierten Neutronen betrug $2 \cdot 10^{7}$.

Die Aussichten für die Erzeugung intensiver Neutronenblitze nach der Hohlpinchmethode haben sich durch die von Becker, KLINGELHÖFER und LOHSE ${ }^{6}$ aufgezeigte Möglichkeit zur gerichteten Einführung von Deuterium und Tritium mit Hilfe kondensierter Molekularstrahlen wesentlich verbessert. In der vor-

4 J. G. Linhart, Nuovo Cimento 17, 850 [1960] ; Nucl. Fusion 1, 78 [1961].

5 Ch. Maisonnier, M. Haegi u. J. G. Linhart, CN 21/79 Frascati; Plasma Phys. and Thermonucl. Reactions, Proc. of Culham Conf., Vol. II, S. 345 [1965], siehe dort auch weitere Literaturangaben.

${ }^{6}$ E. W. Becker, R. Klingelhöfer u. P. Lohse, Z. Naturforsch. 15 a, 645 [1960]. Die Verwendbarkeit kondensierter Molekularstrahlen bei Hohlpinch-Experimenten wurde inzwischen experimentell mit Stickstoff-Clustern nachgewiesen, vgl. E. W. BeCKer, H. Burghoff u. R. KlingelHÖFer, Z. Naturforsch. 22 a, 589 [1967]. 
liegenden Arbeit ${ }^{7}$ wird daher theoretisch untersucht, welche Neutronenblitzrate bei einem mit kondensierten D-T-Molekularstrahlen durchgeführten Hohlpinchexperiment zu erwarten ist.

Die von LINHART und Mitarbeitern durchgeführten Rechnungen 4, 5,8 zur Beschreibung des Hohlpinchvorganges benutzten die vereinfachende Voraussetzung verschwindender Schichtdicke des Hohlzylinders. Mit dieser Näherung läßt sich zwar ungefähr berechnen, welcher Anteil der im Kondensator gespeicherten Energie in kinetische Energie der radial beschleunigten Plasmaschicht verwandelt wird, nicht hingegen, welchen Enddurchmesser und damit welche Enddichte das Plasma erreicht. Um dennoch zu einer Aussage über diese Größen sowie zu einer Abschätzung der Reaktions- bzw. Neutronenrate zu kommen, ging Linhart davon aus, da $\beta$ sich in der beschleunigten Plasmaschicht eine Boltzmann-Verteilung der Dichte einstellt, deren $e$-tel Wertsbreite, die sich während der radialen Beschleunigung einstellt, als $\mathrm{Ma} ß$ für die Schichtdicke angenommen wurde.

In der vorliegenden Arbeit wurde das Differentialgleichungssystem für die Beschleunigung der Schicht durch einen Druckterm ergänzt, so daß sich die Dichte und die Temperatur des Plasmas und damit die emittierte Neutronenrate zu jedem Zeitpunkt des Kollabiervorganges berechnen lassen. Die Bedingungen für maximale Neutronenproduktion werden angegeben. Bei einer in der Kondensatorbatterie gespeicherten Energie von $200 \mathrm{~kJ}$ sind Momentanraten von mehr als $10^{22}$ Neutronen pro Sekunde für einige nsec, d. h. fast $10^{14}$ Neutronen pro Entladung zu erwarten.

\section{Formulierung der Differentialgleichungen}

Das Schema der Hohlpinchanordnung ist in Abb. 1 wiedergegeben. Für die Rechnung wird angenommen, daß der Hohlzylinder sich von Anfang an wie ein vollionisiertes Plasma verhält. Auf die vom Strom durchflossenen Volumenelemente der Hohlzylinderschicht wirkt eine Lorentz-Kraft, die mit der Magnetfeldstärke monoton von der äußeren Oberfläche auf Null an der inneren Oberfläche abnimmt. Dadurch werden die äußeren Massenelemente stär-

7 R. G. A. Frank, Berechnung der Neutronenemission eines mit einer Hohlpinch-Entladung erzeugten D-T-Plasmas (Dissertation), KFK 992 [1969].

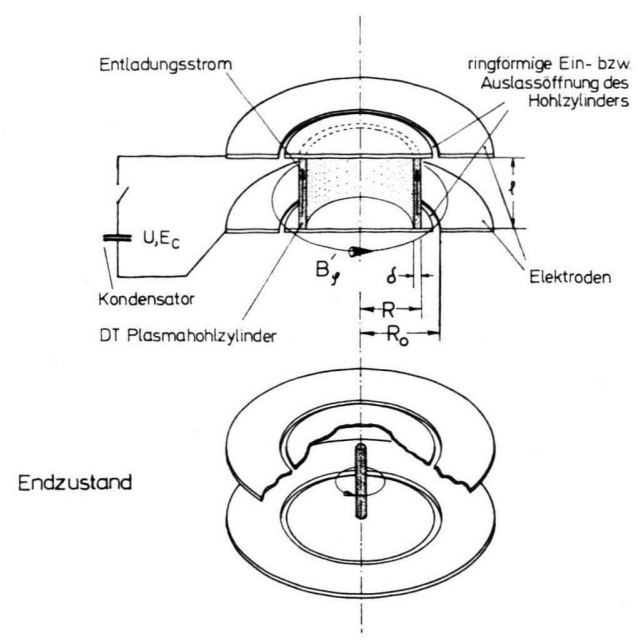

Abb. 1. Versuchsanordnung des Hohlpinches während der Kollabierphase und im Endzustand. $C=$ Kondensatorkapazität ; $U=$ Ladespannung, $R=$ Hohlzylinderradius ; $\delta=$ Schichtdicke; $l=$ Hohlzylinderhöhe.

ker beschleunigt als weiter innen liegende. Das führt zu einer Kompression und adiabatischen Aufheizung der gesamten Schicht, bis sich eine Boltzmann-Verteilung der Dichte in der Schicht entsprechend der barometrischen Höhenformel einstellt, wobei an Stelle der Gravitationsfeldstärke $g$ die Beschleunigung der Schicht steht. Eine strenge mathematische Beschreibung müßte daher die radialen Abhängigkeiten von Lorentz-Kraft und Dichte berücksichtigen, was zu einer partiellen Differentialgleichung führen würde. Um diese Schwierigkeit zu umgehen, wird näherungsweise angenommen, $\mathrm{da} \beta$ die gesamte in der Schicht enthaltene Masse durch die Lorentz-Kraft an der äußeren Oberfläche beschleunigt wird. Das gilt um so besser, je kleiner die Schichtdicke gegenüber dem Radius ist.

Mit dieser Näherung läßt sich eine gewöhnliche Differentialgleichung formulieren, die die Bewegung der Hohlzylinderschicht beschreibt:

$$
\begin{aligned}
N m_{\mathrm{i}} \frac{\mathrm{d} u}{\mathrm{~d} t}= & -\frac{\mu_{0}}{4 \pi} \frac{J^{2}}{R} \\
& +N\left(k T_{\mathrm{e}}+k T_{\mathrm{i}}\right) \frac{1}{F} \frac{\mathrm{d} F}{\mathrm{~d} t} \frac{1}{u} ;
\end{aligned}
$$

$N=$ die Zahl der Ionen pro Längeneinheit; $m_{\mathrm{i}}=$ Ionenmasse; $u=$ Geschwindigkeit der Hohlzylinderschicht; $F=$ Querschnittsfläche des Hohlzylinders; $J=$ Entladungsstrom; $k T_{\mathrm{e}, \mathrm{i}}=$ thermische Elektro-

8 Ch. Maisonnier, J. G. Linhart u. M. Haegi, Nucl. Fusion 2, Suppl. Part 2, 727 [1962]. - M. Bineau u. Ch. MaisonNIER, Gas Discharges and Electr. Suppl. Industry, Butterworth, London 1962, S. 517-528. 
nen- bzw. Ionenenergie. Es ist $N=F \cdot n=$ const, $n=$ Ionendichte. Der erste Term der rechten Seite ist die Lorentz-Kraft pro Längeneinheit, der zweite die Kraft pro Längeneinheit auf Grund des gaskinetischen Druckes der Ionen und Elektronen.

Die zeitliche Änderung der thermischen Elektronen- bzw. Ionenenergie ist gleich der pro Zeiteinheit geleisteten adiabatischen Expansions- bzw. Kompressionsarbeit. Die thermische Energie der Elektronen wird darüber hinaus durch Ohmsche Aufheizung erhöht. Das führt zu einer Differenz der thermischen Energien von Elektronen und Ionen, die mit der Rate $R^{\text {ei }}$ ausgeglichen wird:

$$
\begin{gathered}
\frac{\mathrm{d}}{\mathrm{d} t} k T_{\mathrm{e}}=-\frac{2}{3} k T_{\mathrm{e}} \frac{1}{F} \frac{\mathrm{d} F}{\mathrm{~d} t}-\frac{2}{3} \frac{R^{\mathrm{ei}}}{n}+\frac{2}{3} \frac{\eta(J / F)^{2}}{n} ; \\
\frac{\mathrm{d}}{\mathrm{d} t} k T_{\mathrm{i}}=-\frac{2}{3} k T_{\mathrm{i}} \frac{1}{F} \frac{\mathrm{d} F}{\mathrm{~d} t}+\frac{2}{3} \frac{R^{\mathrm{ei}}}{n} .
\end{gathered}
$$

Der spezifische Widerstand $\eta^{9}$ nimmt mit wachsender Elektronentemperatur $\mathrm{ab}$ :

$$
\eta=\frac{4}{3} \sqrt{2 \pi} m_{\mathrm{e}}{ }^{1 / 2} e^{2} \frac{\ln A}{\left(k T_{\mathrm{e}}\right)^{3 / 2}},
$$

wobei

$$
\Lambda=\frac{3}{2 e^{3}} \frac{\left(k T_{\mathrm{e}}\right)^{3 / 2}}{\sqrt{\pi n}} ;
$$

$m_{\mathrm{e}}=$ Elektronenmasse; $e=$ Elementarladung. Da die Ohmsche Aufheizung mit wachsender Elektronentemperatur stark abnimmt, stellt sich nach Beginn der Entladung schnell eine Elektronentemperatur ein, die vom gewählten Anfangswert nahezu unabhängig ist, solange dieser Anfangswert nicht größer als einige $\mathrm{eV}$ ist. Die Rate $R^{\mathrm{ei}}$ ist gegeben durch:

$$
R^{\mathrm{ei}}=4 \sqrt{2 \pi} \frac{n^{2} m_{\mathrm{e}} \mathrm{e}^{1 / 2} e^{4}}{m_{\mathrm{i}}} \ln \Lambda \frac{k T_{\mathrm{e}}-k T_{\mathrm{i}}}{\left(k T_{\mathrm{e}}\right)^{3 / 2}} .
$$

Die Querschnittsfläche $F$ des Hohlzylinders ist:

$$
F=2 \pi R \delta(1-\delta / 2 R) .
$$

Damit wird die relative, zeitliche Änderung der Fläche $F$ gleich:

$$
\frac{1}{F} \frac{\mathrm{d} F}{\mathrm{~d} t}=\frac{1}{1-\delta / 2 R}\left\{\frac{u}{R}+\left(1-\frac{\delta}{R}\right) \frac{1}{\delta} \frac{\mathrm{d} \delta}{\mathrm{d} t}\right\} .
$$

Sobald der Hohlzylinder durch Zusammenschnüren zum Vollzylinder verschmilzt, wird:

$$
F=\pi R^{2}
$$

${ }^{9}$ L. SpITzer, Physics of Fully Ionized Gases, Interscience Publishers, Inc., New York 1956. und die relative, zeitliche Änderung:

$$
\frac{1}{F} \frac{\mathrm{d} F}{\mathrm{~d} t}=2 \frac{u}{R} .
$$

Mit der zeitlich veränderlichen Induktivität $L$ des Entladekreises:

$$
L=L_{\mathbf{0}}+\left(\mu_{0} / 2 \pi\right) l \ln \left(R_{0} / R\right)
$$

wird die Stromstärke $J$ durch folgende Differentialgleichung bestimmt:

$$
\begin{gathered}
\frac{\mathrm{d} J}{\mathrm{~d} t}=-\frac{1}{L_{0}-\frac{\mu_{0}}{2 \pi} l \ln \left(R / R_{0}\right)} \cdot\left\{\left(\frac{\eta l}{F}-\frac{\mu_{0}}{2 \pi} l \frac{u}{R}\right) J+\frac{Q}{C}\right\} \\
Q=\text { Kondensatorladung. }
\end{gathered}
$$

Mit zunehmender Leitfähigkeit $1 / \eta$ und kleiner werdendem Radius $R$ wird der Ohmsche Widerstand vernachlässigbar klein gegenüber dem induktiven Widerstand.

Die Bewegungsgleichung (1) reicht allerdings nicht aus, beide Größen, den Radius $R$ und die Schichtdicke $\delta$, als Funktion der Zeit zu bestimmen. Infolgedessen muß durch zusätzliche Annahmen ein physikalisches Modell formuliert werden, aus dem sich die Schichtdicke ergibt.

Die Lösung des Differentialgleichungssystems ist in analytischer Form nicht möglich. Es wurde daher ein numerisches Integrationsverfahren für die IBM 7070-Rechenanlage des Kernforschungszentrums Karlsruhe entwickelt, mit dem sich die Lösungen unter Vorgabe der die Hohlpinchanordnung festlegenden Parameter errechnen lassen. Das entwikkelte Hohlpinchmodell soll im folgenden an Hand eines Lösungsbeispieles erläutert werden.

\section{Lösungsbeispiel}

Der in Abb. 2 skizzierte Verlauf der Variablen liegt den folgenden Parametern einer Hohlpinchanordnung zugrunde. Die Abmessung des Hohlzylinders ist gegeben durch den Anfangsradius $R_{0}=30$ $\mathrm{cm}$, die Höhe $l=10 \mathrm{~cm}$ und die Anfangsschichtdicke $\delta_{0}=0,5 \mathrm{~cm}$. Das Plasma im Hohlzylinder besitzt eine Anfangsdichte $n_{0}=7,1 \cdot 10^{16}$ Ionen $/ \mathrm{cm}^{3}$ und eine Anfangstemperatur $k T_{\mathrm{e}}=1 \mathrm{eV}$ und $k T_{\mathrm{i}}=0,95 \mathrm{eV}$. In der angeschlossenen Kondensatorbatterie ist eine Energie von $200 \mathrm{~kJ}$ bei einer Ladespannung $U=$ $100 \mathrm{kV}$ und einer Kapazität $C=40 \mu \mathrm{F}$ gespeichert. Die Induktivität $L_{0}$ wurde zu $10 \mathrm{nH}$ abgeschätzt.

In Abb. 2 sind über der Zeit aufgetragen a) der Radius $R$ der äußeren Oberfläche und im Abstand $\delta$ 
von $R$ die innere Oberflächenbegrenzung, b) die kinetische Energie der Ionen $E_{\text {kin }}$, sowie die thermischen Energien von Elektronen $k T_{\mathrm{e}}$ und Ionen $k T_{\mathrm{i}}$, c) die Stromstärke $J$ und die Kondensatorladung $Q$.

a)

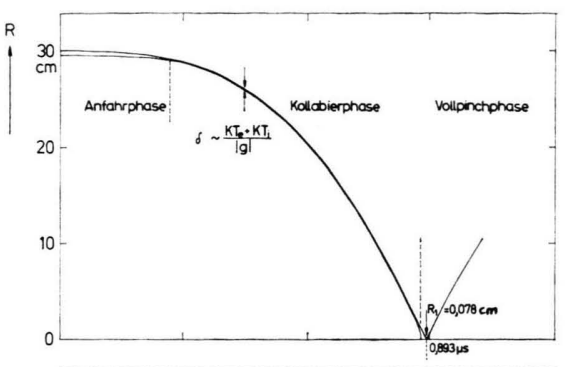

b)

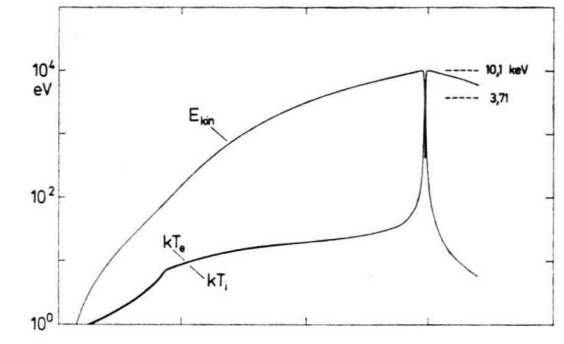

c)

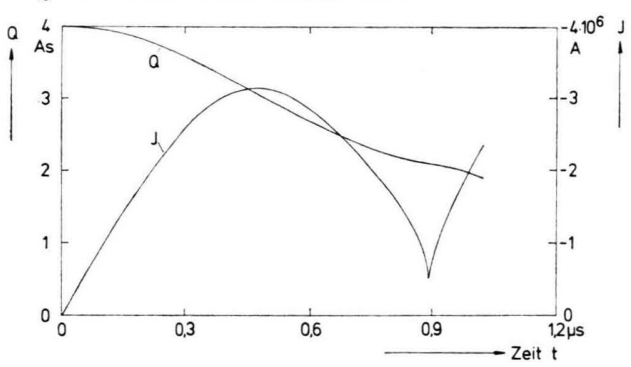

Abb. 2. Der zeitliche Verlauf: a) des den Hohlzylinder begrenzenden äußeren bzw. inneren Radius; b) der kinetischen Energie $E_{\text {kin }}$ der Ionen in der Plasmaschicht und der thermischen Elektronen- und Ionenenergie $\left.k T_{\mathrm{e}, \mathrm{i}} ; \mathrm{c}\right)$ der elektrischen Stromstärke $J$ und der Ladung $Q$ im Kondensator.

Die Schichtdicke wird in der Anfahrphase (Abb. 2 a) durch folgendes Modell bestimmt. Als Folge der Ohmschen Aufheizung der Elektronen würde bei Beginn der Entladung die Schicht nach außen und innen thermisch expandieren. Die Expansion der äußeren Oberfläche wird mit steigender Stromstärke über die Lorentz-Kraft bereits nach 0,063 $\mu \mathrm{sec}$ aufgehalten, so daß die Oberfläche anschließend radial nach innen beschleunigt wird. Die innere Oberfläche hingegen bewegt sich ungehindert in Richtung Zylinderachse mit thermischer Geschwindigkeit, bis die Geschwindigkeit der äußeren Oberfläche größer wird als die der inneren, wobei die Schicht komprimiert wird. Unter Berücksichtigung der Relativge- schwindigkeit läßt sich mit der thermischen Geschwindigkeit die Änderungsgeschwindigkeit der Schichtdicke zu

$$
\frac{\mathrm{d} \delta}{\mathrm{d} t}=\left(1-\frac{\delta}{2 R}\right) u+\left[\frac{2}{3}\left(k T_{\mathrm{e}}+k T_{\mathrm{i}}\right) / m_{\mathrm{i}}\right]^{1 / 2}
$$

angeben. Die Diff.-Gl. (11) legt in der Anfahrphase die Schichtdicke $\delta$ zusammen mit den bereits formulierten Differentialgleichungen fest.

Sobald sich die weiter oben beschriebene Gleichgewichtsverteilung der Dichte in der Schicht eingestellt hat, wird als Schichtdicke die $e$-tel Wertsbreite der Boltzmann-Verteilung angenommen:

$$
\delta=\left(k T_{\mathrm{e}}+k T_{\mathrm{i}}\right) / m_{\mathrm{i}}|\mathrm{d} u / \mathrm{d} t| .
$$

Die Schichtdicke wird in der sich nach $0,27 \mu$ sec an die Anfahrphase anschließenden Kollabierphase durch (12) bestimmt und in die Differentialgleichungen eingesetzt.

In der Vollpinchphase (Innenradius des Plasmahohlzylinders $=0$ ) wird die kinetische Energie, die die Ionen auf Grund ihrer gerichteten Bewegung haben, praktisch vollständig in ungeordnete thermische Energie umgewandelt. Dabei werden Elektronen und Ionen gleichermaßen aufgeheizt. Temperaturunterschiede werden durch Stöße, die durch den Term $R^{\text {ei }}$ beschrieben werden, fast vollständig ausgeglichen. Bereits 12 nsec nach Einsetzen der Vollpinchphase ist praktisch die gesamte in der radialen Bewegung enthaltene Energie in thermische umgewandelt. Der kleinste Durchmesser dieses Endzustandes ist $2 R_{1}=0,156 \mathrm{~cm}$, was eine maximale Dichte von $3,5 \cdot 10^{22}$ Ionen $/ \mathrm{cm}^{3}$ ergibt. Die höchste thermische Ionenenergie in diesem Zustand ist $k T_{\mathrm{i}}=3,71 \mathrm{keV}$. Während der Vollpinchphase wird aus dem zeitlichen Verlauf von Ionentemperatur und Dichte die Neutronenrate ermittelt:

$$
\alpha=\frac{1}{4} n^{2}(\overline{\sigma v}) \quad \text { Neutronen } / \mathrm{cm}^{3} \mathrm{sec} .
$$

Dabei ist $\sigma$ der Wirkungsquerschnitt der D - T-Reaktion und $v$ die relative Geschwindigkeit von $\mathrm{D}$ - und T-Ionen. In Formel (13) steht der über die MaxwellVerteilung der thermischen Ionenenergie gemittelte Wert $(\overline{\sigma v})$. Die Neutronenrate liefert - integriert über das Reaktionsvolumen und die Zeit - die Neutronenausbeute $N_{\mathrm{p}}$. Innerhalb von ca. 1 nsec wird so ein Neutronenblitz von $N_{\mathrm{p}}=2,3 \cdot 10^{13} \mathrm{Neu}$ tronen erzeugt bei einer Reaktionsrate, deren Maximum $\alpha=1,2 \cdot 10^{23}$ Neutronen $/ \mathrm{cm}^{3} \mathrm{sec}$ ist. 


\section{Optimalisierung der Versuchsparameter}

Bei vorgegebener Kapazität $C$ und Ladespannung des Kondensators gibt es eine optimale Ionenzahl im Plasmahohlzylinder, bei der die Neutronenausbeute ein Maximum durchläuft, was die Betrachtung zweier Grenzfälle plausibel macht. Bei beliebig zunehmender Ionenzahl geht die Energie pro Ion und damit der Wirkungsquerschnitt für die D-T-Reaktion gegen Null und bei beliebig abnehmender Ionenzahl geht die Anzahl der reagierenden Teilchen gegen Null. Die zu jedem Radius $R_{0}$ gehörende optimale Ionenzahl wurde für drei Werte des Energieinhaltes der Kondensatorbatterie ausgerechnet, und die entsprechenden Neutronenausbeuten sind in Abb. 3 über dem Anfangsradius $R_{0}$ aufgetragen.

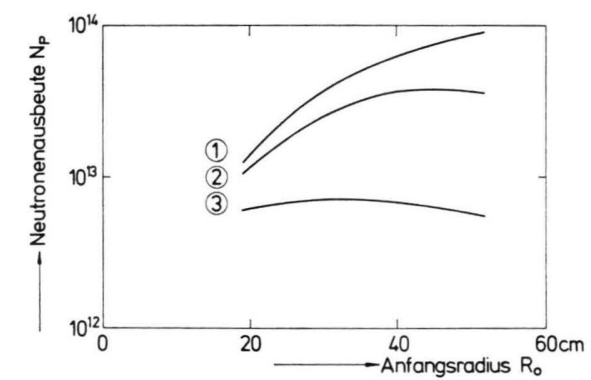

Abb. 3. Die zu jedem Anfangsradius $R_{0}$ maximale Neutronenausbeute $N_{\mathrm{p}}$. Parameter der Kurven 1, 2 und 3 ist die Kondensatorenergie $E_{\mathrm{c}}=300,200$ und $100 \mathrm{~kJ}$.
Da sich mit wachsendem $R_{0}$, d. h. größer werdender Beschleunigungsstrecke ein zunehmender Anteil der gespeicherten Energie als kinetische Energie auf das Plasma übertragen läßt, erwartet man zunächst, daß $N_{\mathrm{p}}\left(R_{0}\right)$ gegen einen Grenzwert geht, wenn die gesamte gespeicherte Energie in das Plasma überführt ist.

Es stellt sich jedoch heraus, daß die Thermalisierungszeiten der Ionen wegen der Abnahme des Coulomb-Stoßquerschnittes mit wachsender Energie pro Ion, d. h. mit steigendem $R_{0}$, länger werden als die Zeit während der Vollpinchphase, in welcher der Hauptanteil der zu $N_{\text {p }}$ beitragenden Reaktionen stattfindet. Um die - verglichen mit nicht thermalisierten Ionen - relativ hohe Reaktionsrate thermalisierter Ionen ausnutzen zu können, ist man gezwungen, durch Vergrößerung der Ionenzahl und damit Verkleinerung der Ionenenergie die Thermalisierungszeit künstlich zu verkürzen. Dadurch wird die Neutronenausbeute gedrückt, und zwar, wie die Analyse zeigt, mit wachsendem $R_{0}$ so stark, daß $N_{\mathrm{p}}$ ein Maximum durchläuft. Es ist plausibel, daß sich dieses Maximum mit steigender Kondensatorenergie zu höheren Werten von $R_{0}$ und $N_{\mathrm{p}}$ verschiebt.

Herrn Professor Dr. E. W. Becker danken wir für stete Förderung und das der Arbeit entgegengebrachte Interesse. 